\title{
Un modelo basado en agentes para simular la capacidad de absorción en organizaciones
}

\author{
Orlando López-Cruz ${ }^{1}$ \\ saudiel.lopez@javeriana.edu.co \\ ${ }^{1}$ Pontificia Universidad Javeriana, Calle 147 14-69 T.5-704, Bogotá D.C. 110121, Colombia.
}

DOI: 10.17013/risti.26.122-139

\begin{abstract}
Resumen: Se presenta una re-conceptualización del constructo 'capacidad de absorción' (ACAP) en las organizaciones que pretende ser implementable en un programa de ordenador para facilitar la simulación de los efectos de la adquisición, asimilación y explotación de conocimiento aprovechable del entorno de la organización, con el objetivo de generar ventaja competitiva e innovación en las organizaciones. A diferencia de otras investigaciones que se enfocan en producir refinamientos al constructo de la ACAP, ésta investigación se centra en una simulación por ordenador para diseñar la ACAP real en las organizaciones. Se sugiere de una falta de diseño en los modelos conceptuales inferidos de la revisión de la literatura y se propone un nuevo diseño implementable de la ACAP. Este nuevo diseño arroja luces sobre la implementación en el entorno organizacional de la ACAP capaz de sustentar la absorción efectiva de conocimiento en las organizaciones.
\end{abstract}

Palabras-clave: Capacidad de absorción; gestión de conocimiento; innovación tecnológica, modelos basados en agentes.

\section{Anagent-based model to simulate absorption capacity in organizations}

\begin{abstract}
This paper introduces a reconceptualization of the 'absorptive capacity' (ACAP) construct in organizations pursuing to implement a computer program to assist the simulation the results of acquisition, assimilation and harnessing of knowledge available in the environment of the organization, with the aim of obtaining competitive advantage and produce innovation in organizations. In contrast to other researches focusing in refining the ACAP construct, this research emphasizes on a computer simulation to assist the design an actual ACAP in organizations. This paper suggests a lack of design of the ACAP conceptual models inferred from the reviewed literature and a new implementable design of the ACAP construct is proposed. This new design sheds light upon an actual implementation of the ACAP construct able to support effective knowledge absorption at organizations.
\end{abstract}

Keywords: Absorptive capacity; knowledge management, technological innovation; agent-based models. 


\section{Introducción}

El constructo 'capacidad de absorción' (abreviado como ACAP en alguna literatura en inglés por Absorptive Capacity) está relacionado con la visión de las organizaciones basada en el conocimiento (Knowledge-Based View-KBV) (Kathleen M Eisenhardt \& Santos, 2002; Grant, 1996; Nickerson \& Zenger, 2004), con el concepto de conocimiento en la teoría del aprendizaje organizacional (Argyris \& Schön, 1978; Levitt \& March, 1988) y con la adopción de sistemas de gestión del conocimiento desde el enfoque del receptor (León-Sigg, Vázquez-Reyes, \& Villa-Cisneros, 2017; Palos-Sánchez, ArenasMárquez, \& Aguayo-Camacho, 2017; Sousa, Costa, \& Aparicio, 2017). La finalidad de esta conceptualización es encontrar la forma de generar ventaja competitiva en las organizaciones (Porter, 1998), a partir de conocimiento codificado o no (explícito o tácito) (Nonaka \& Takeuchi, 1995; Nonaka, Toyama, \& Nagata, 2000). Puesto que la visión basada en el conocimiento trata de explicar el desempeño de las empresas (Grant, 1996) con base en el conocimiento explotable, lo crítico es hallar la forma como el conocimiento puede apropiarse y explotarse para hacer sostenible la ventaja competitiva alcanzada.

Esta investigación presenta (i) el re-diseño del constructo de la ACAP que permite describir tanto la adquisición como la explotación del conocimiento con fines de sostener ventaja competitiva y (ii) los resultados de la implementación de dicho diseño en un programa de ordenador en un sistema basado en agentes orientado a la simulación interactiva con usuarios expertos.

Este documento se estructura de la siguiente forma: se presenta el constructo de la ACAP y el concepto de 'rutina organizacional' para luego relacionarlos, la metodología utilizada y luego se presenta la propuesta de re-diseño del constructo, su implementación y la presentación de resultados y análisis.

\section{El constructo "capacidad de absorción"}

El constructo "capacidad absorbente" o "capacidad de absorción" fue extendido, en la década de los 1990’s (Cohen \& Levinthal, 1990), desde el dominio de la hidrología, la ecología y la medicina, al campo de las organizaciones como una capacidad dinámica de estos sistemas sociales humanos complejos. En ese sentido, es un concepto inspirado en sistemas complejos naturales.

Este constructo, 'capacidad de absorción' (Cohen \& Levinthal, 1990), entendido como una medida de la aptitud de una organización (Helfat et al., 2009; D. Teece \& Pisano, 1994; D. J. Teece, Pisano, \& Shuen, 1997) para (i) escrutar su entorno y reconocer el valor de conocimiento externo nuevo, adquirirlo, (ii) asimilarlo, (iii) transformarlo, explotarlo con sus recursos internos y que le sirva como marco para la innovación, es una variable crítica para caracterizar las posibilidades para que haya una efectiva transferencia de conocimiento tecnológico a la organización sobre la que se hace la medición, proceso similar al de la elicitación de requerimientos, que es intensivo en la transferencia de información entre los que son «poseedores del conocimiento» desde el lado del cliente-usuario y un equipo de desarrollo de software (Donoso Barraza \& Vega Zepeda, 2017). 


\subsection{Origen y desarrollo del constructo ACAP.}

El constructo 'capacidad de absorción' ACAP (por su apócope en inglés de Absorptive Capacity) fue originalmente definido con una habilidad basada en el conocimiento previo (Cohen \& Levinthal, 1990) para reconocer el valor de nueva información, asimilarla y aplicarla para propósitos comerciales. Esta propuesta de ACAP es altamente conceptual y, por lo mismo, conduce a un modelo que es difícilmente implementable en modelos por ordenador.

Se proponen cuatro periodos cronológicos del desarrollo del constructo 'capacidad de absorción'.

El primer periodo es la aparición del constructo, el segundo periodo es el de fundamentación conceptual que ha permitido conformar un dominio del conocimiento. El hito de inicio de este periodo es la aparición de la primera re-conceptualización de la capacidad de absorción como una "capacidad dinámica" (Zahra \& George, 2002). En un tercer periodo, se consolida la investigación empírica de capacidad de absorción en business research (Lewin, Massini, \& Peeters, 2011) relacionándose con la investigación sobre ventaja competitiva, transferencia de tecnología (patentes e invenciones), aprendizaje organizacional y, finalmente, en el más reciente periodo se extiende el dominio de investigación de la capacidad de absorción a la ingeniería de organizaciones, se estrechan las relaciones con los conceptos de transferencia de conocimiento, sistemas basados en conocimiento (base de conocimiento + motores de inferencia o sistemas de razonamientos simbólico), adquisición de conocimiento, representación de conocimiento y capital humano.

El constructo “capacidad de absorción” emergió (Cohen \& Levinthal, 1989, 1990, 1994) para referirse a una capacidad dinámica (Helfat et al., 2009; Prahalad \& Hamel, 2006; D. Teece \& Pisano, 1994; D. J. Teece et al., 1997) de una organización expresada en tres dimensiones, capacidad para:

i. identificar o reconocer,

ii. asimilar y

iii. aplicar o aprovechar el conocimiento disponible en el entorno.

A inicios del siglo XXI, la capacidad de absorción fue re-conceptualizada (Zahra \& George, 2002) como el conjunto de rutinas organizacionales y procesos por los cuales las organizaciones:

i. adquieren,

ii. asimilan,

iii. transforman y

iv. explotan

el conocimiento para producir una capacidad dinámica (Helfat et al., 2009; Prahalad \& Hamel, 2006; D. Teece \& Pisano, 1994; D. J. Teece et al., 1997).

Una organización cuenta con capacidades organizacionales operacionales (sustantivas) y dinámicas (Winter, 2000). Una capacidad organizacional es una rutina de alto nivel que, con procesos y recursos específicos de la organización, le confiere a sus directivos 
un conjunto de opciones de decisión para producir resultados significativos de una clase particular, usualmente el logro de metas estratégicas, utilizando el know-how disponible y recursos no específicos de la organización (D. J. Teece, 1977; D. J. Teece et al., 1997; Winter, 2000). Una capacidad operacional se refiere a competencias y habilidades que determinan la efectividad de una organización para ejecutar sus actividades misionales cotidianas. Una capacidad dinámica organizacional es la capacidad para integrar, construir y reconfigurar capacidades operativas (D. J. Teece et al., 1997), así como competencias internas y externas para afrontar cambios rápidos del entorno (Zollo \& Winter, 2002). Está orientada hacia el cambio organizacional, por lo que permite formular una respuesta apropiada e implementar un curso de acción de la organización, reflejando su carácter evolutivo.

Una nueva reconceptualización (Todorova \& Durisin, 2007) retoma la dimensión de (i) identificar o reconocer el valor del conocimiento, separa la (ii) adquisición de la (iii) asimilación y deja esta última como una etapa o dimensión alternativa frente a la (iv) transformación de conocimiento, previa a la (iv) explotación (aplicación o aprovechamiento) del conocimiento. Además, quizás lo más valioso, es la inclusión de un ciclo de retroacción (feedback) que hace coherente al constructo con la dinámica que no puede ser capturada por los métodos analíticos (Todorova \& Durisin, 2007), lo que revela una oportunidad de seleccionar una metodología de investigación apropiada a la investigación en ingeniería.

En una perspectiva de categorización, los resultados de los estudios sobre ACAP pueden ser clasificados en: identificación de los componentes del constructo ACAP (González, 2014; Nishiyama, Ikeda, \& Niwa, 2000; Todorova \& Durisin, 2007; Vega, Gutiérrez, \& Fernández de Lucio, 2007), el papel de las condiciones contextuales y otros factores externos relacionados con la innovación en la organizaciones (Fabrizio, 2009) y sus aplicaciones (López-Paniagua, Nieto-Carlier, Rodríguez-Martín, González-Fernández, \& Jiménez-Álvaro, 2011; Volberda, Foss, \& Lyles, 2009), diseño de instrumentos de medición (Marín, Laureiro, \& Forero, 2007), investigación experimental (Filgueiras Sainz de Rozas, Castro Fernández, \& Rafull Suárez, 2013) y su relación con la transferencia de tecnología (López-Cruz, 2010).

\subsection{El constructo ACAP y las rutinas organizacionales.}

Pasados doce años de la publicación del artículo seminal (Cohen \& Levinthal, 1990), el constructo ACAP fue re-conceptualizado en forma importante (Zahra \& George, 2002) como un conjunto de rutinas organizacionales. Es decir, la ACAP es evocada como una capacidad dinámica que amplía las habilidades organizacionales para obtener y sostener la ventaja competitiva (Porter, 1998) y para generar cambios organizacionales de manera estratégica.

Al comparar ésta propuesta (Zahra \& George, 2002) con el constructo original (Cohen \& Levinthal, 1990), la ACAP se transforma en (i) una capacidad dinámica, (ii) las rutinas organizacionales son los componentes principales de la ACAP y (iii) se identifican condiciones bajo las cuales los componentes de la ACAP son capaces de crear valor. Esto permite conducir investigaciones sobre las diferencias entre dos organizaciones que operan en el mismo sector económico y, más importante aún, prescribir esas diferencias. 


\subsection{El constructo ACAP y las capacidades organizacionales.}

Una capacidad organizacional es una rutina de alto nivel que, por medio de procesos y recursos específicos organizacionales, proveen a la alta gerencia elementos de juicio para tomar decisiones que producen resultados significativos de alguna clase en particular. Usualmente, estos resultados son metas estratégicas para usar conocimiento (know-how) disponible y recursos organizacionales no-específicos (D. J. Teece, 1977; Winter, 2000).

Las capacidades organizacionales proceden de tres factores:

i. recursos,

ii. procesos y

iii. valores (Christensen \& Overdorf, 2000).

De las capacidades organizacionales, que pueden ser capacidades operacionales (sustantivas) y capacidades dinámicas, las capacidades dinámicas se originan, entre otras fuentes, en las estructuras de la organización (Easterby-Smith, Graca, Antonacopoulou, \& Ferdinand, 2005) lo cual implica que las capacidades dinámicas están determinadas funcionalmente por variables de la estructura de la organización. Es decir, las variables de la estructura de la organización determinan las capacidades dinámicas. Esto no implica que sean las únicas variables que determinan las capacidades dinámicas, puesto que se ha identificado a partir de otros estudios que no hay una relación causa efecto entre las variables de estructura de la organización y las capacidades dinámicas (Dozier \& Chang, 2009). Además, al parecer tampoco hay una relación unidireccional causaefecto entre las variables de estructura organizacionales y las capacidades dinámicas (Cordes-Berszinn, 2013).

Puesto que la ventaja competitiva de una organización (Porter, 1998) se fundamenta en sus capacidades dinámicas (D. Teece \& Pisano, 1994), entonces las organizaciones necesitan desarrollarlas (Giovanni Dosi \& Marengo, 2000; Kathleen M. Eisenhardt \& Martin, 2000; D. J. Teece et al., 1997; Zollo \& Winter, 2002) para adaptarse a su entorno dinámico.

Lo esencial de las competencias y las capacidades es que no pueden ser adquiridas en el mercado, de la misma forma que las propiedades internas de las organizaciones no pueden ser replicadas como un conjunto de unidades de negocio (a través de contratos formales en el mercado). Las capacidades organizacionales deben ser entendidas tanto en términos de estructuras organizacionales como de procesos administrativos (D. J. Teece et al., 1997 p.517) y no en términos del balance general, o de los estados financieros en general.

Los componentes de la ACAP -las rutinas organizacionales- (Bobrow \& Winograd, 1977) - se agrupan en dos conjuntos: capacidad de absorción potencial y capacidad de absorción realizada. Las rutinas de ACAP potencial son adquisición de conocimiento y asimilación de conocimiento. Las rutinas de ACAP realizada son transformación y explotación. El objetivo de las rutinas de ACAP potencial es incentivar la receptividad al conocimiento externo, que es débilmente equivalente a la dimensión "reconocer el valor del conocimiento (Cohen \& Levinthal, 1990). Las rutinas de ACAP realizado buscan apalancar el aprendizaje organizacional con base en el conocimiento absorbido.

Los investigadores de la primera década del siglo XXI sugirieron que estas rutinas organizacionales proveían mecanismos sistémicos, estructurales y procedimentales 
para que la organización explotara el conocimiento y obtuviera beneficios de largo plazo pese a las barreras como la 'apropiabilidad' (es decir, las dinámicas institucionales como la propiedad intelectual, que afecta las capacidades organizacionales para proteger sus derechos de beneficiarse de los nuevos productos o procesos). La 'apropiabilidad' del conocimiento está en relación inversa con las propensión organizacional a invertir en investigación y desarrollo (Lane, Koka, \& Pathak, 2006).

Sin embargo, este modelo (Zahra \& George, 2002) exhibe ambigüedades (Todorova \& Durisin, 2007), por ejemplo la omisión de rutinas que en la propuesta original son sólo componentes del constructo para "reconocimiento del valor del conocimiento externo". La literatura advierte (Todorova \& Durisin, 2007) que los modelos analíticos lineales (Cohen \& Levinthal, 1990; Zahra \& George, 2002) no describen en forma apropiada la complejidad de las relaciones causales de la ACAP que explican la dinámica de absorción a través de los procesos de aprendizaje organizacional (Lane et al., 2006).

Un nuevo modelo de la ACAP (Todorova \& Durisin, 2007) (i) retoma el componente de identificación del valor del conocimiento (Cohen \& Levinthal, 1990), extiende el alcance de la incidencia (bien sea positiva o negativa) de los mecanismos sociales de integración (Easterby-Smith et al., 2005) a todas las rutinas organizacionales teorizando los factores de contingencia, (iii) incluye en el conjunto de factores de contingencia a las relaciones de poder que influencian tanto a las rutinas de valoración del conocimiento, como a las rutinas de explotación del conocimiento, (iv) reconoce las relaciones complejas entre las dimensiones de asimilación y transformación, (v) redefine las rutinas de transformación y (vi) incluye un ciclo de retroacción que caracteriza la dinámica y complejidad del fenómeno.

Desde este punto de vista, la organización puede ser concebida como un repositorio de conocimiento. Una organización puede ser modelada como un instrumento para transferir y desarrollar conocimiento no sólo a otras organizaciones con las que se relacione (es decir, no sólo para crear y aplicar conocimiento, que es una visión estática), sino que se expone a un flujo de conocimiento. De aquí que, la ACAP parece ser el constructo más apropiado para explicar la forma como las organizaciones pueden realmente estar en capacidad de adquirir conocimiento y hacerlo productivo. Esto es importante porque estudios previos indican que la transferencia de conocimiento depende del nivel de la ACAP como también de la complejidad del conocimiento que está siendo transferido y de los aspectos sociales en entorno turbulentos (Dhanaraj, Lyles, Steensma, \& Tihanyi, 2004).

Una estructura que se enfoca en la gestión del conocimiento (Huber, 1991) se despliega en cuatro procesos de aprendizaje: (1) adquisición de conocimiento, (29 distribución de información, (3) interpretación de información y (4) memoria organizacional. Esta estructura de procesos semeja y complementa el constructo de la ACAP.

\section{Metodología}

El paradigma de la investigación basada en el diseño se origina en las ciencias de lo artificial (A. Hevner, March, Park, \& Ram, 2004; Simon, 1996), entre las que se cuenta la ingeniería. La finalidad prioritaria de las ciencias de la ingeniería no es crear teorías de validez universal, describir, entender, comprender, predecir o explicar la naturaleza de los fenómenos (lo que es). Todo lo anterior sí es prioridad de las ciencias naturales. 
Para las ciencias de lo artificial es objetivo prioritario el diseño, construcción e integración de artefactos al mundo (lo que puede ser) (A. R. Hevner \& Chatterjee, 2010), incrustarlos adecuadamente en la realidad con una finalidad, por lo que el aspecto relevante de las ciencias de lo artificial es la interfaz entre las leyes naturales que gobiernan los entornos interno y externo al artefacto con el fin último de alcanzar logros adaptando las leyes internas a las leyes externas. Por lo anterior, la investigación basada en diseño, en contraste con la investigación en ciencia, está enfocada en cómo deben ser las cosas, lo que advierte elementos deontológicos de este enfoque de investigación, concibiendo artefactos que cumplan con metas (Simon, 1996 p.113-114).

Ahora bien, las metas están asociadas con el espacio de problemas ubicado entre la situación actual y la situación deseada (Simon, 1996). Y en el campo de la investigación de sistemas de información un paso importante es la demostración de que un artefacto de tecnología de información (TI) es o no es adecuado para una un problema especificado, como también lo es la comparación de la utilidad de artefactos TI existentes dentro de contextos organizacionales específicos (March \& Storey, 2008). Tanto los sistemas de información como las rutinas organizacionales son extensiones de las capacidades cognitivas humanas (A. R. Hevner \& Chatterjee, 2010).

La investigación basada en el diseño se realiza en tres ciclos:

i. el ciclo de relevancia, que vincula el entorno contextual del proyecto de investigación con las actividades de diseño: "una buena investigación basada en el diseño empieza por identificar y representar oportunidades en un entorno de aplicación real";

ii. el ciclo de rigor, que conecta las actividades de investigación basada en diseño con la base de conocimiento, la experiencia que provee información al proyecto de investigación, provee el conocimiento previo requerido para el proyecto de investigación y asegurar su innovación, y

iii. el ciclo de diseño, que itera entre las actividades de núcleo de construcción de un artefacto, su evaluación y la retroacción subsecuente para refinar más el diseño (A. R. Hevner \& Chatterjee, 2010).

La construcción del modelo basado en agentes se realizó mediante el protocolo ODD (Grimm et al., 2006; Müller et al., 2013) para separar el proceso de modelado de los detalles de implementación y del lenguaje de programación (Grimm et al., 2006). El modelado basado en agentes es recomendado en entornos en donde se hace imperativo explorar posibles comportamientos o escenarios en el contexto de la incertidumbre (Alexandre \& Balsa, 2018). De esta forma, se elaboró el modelo conceptual de un sistema basado en agentes. En ese modelo, los agentes son organizaciones, el flujo de conocimiento se representa por 'artefactos tecnológicos' de la misma forma que el 'conocimiento' externo a las organizaciones y las barreras organizacionales para acceder al conocimiento. Estos describen el dominio situacional y las especificaciones del modelo para las fases del protocolo ODD (I) Resumen, (II) Conceptos de diseño y (III) Detalles.

El conocimiento es entendido y representado como rutinas organizacionales. Una organización del modelo tiene su propio conjunto de rutinas. Una rutina organizacional tiene acceso completo a los ‘artefactos tecnológicos' y ‘el conocimiento' en su vecindario (tienen la capacidad de identificar conocimiento en el flujo de conocimiento) con el fin de 
decidir si "capturan” o no el conocimiento en el flujo externo. Además, una organización tiene una 'base de conocimiento' que es una lista de tareas para realizar sus actividades. El conocimiento decae en datos y, por eso, desaparece del modelo.

La verificación del modelo incluye la verificación del código en términos de (i) detección de errores y depuración en el programa de ordenar y (ii) evaluación de que el programa implementado corresponda con sus especificaciones. Esto lleva a evaluar si el modelo conceptual ha sido implementado en el lenguaje de programación. Esto se hizo antes del proceso de validación (Sankararaman \& Mahadevan, 2015). La validación del sistema basado en agentes es una tarea difícil cuando se aborda de la misma manera que los modelos científicos tradicionales (Klügl, 2008; Parunak, Savit, \& Riolo, 1998; Xiang, Kennedy, Madey, \& Cabaniss, 2005). En esta investigación, la validación fue realizada por sujetos expertos(Niazi, Hussain, \& Kolberg, 2009) vinculados a organizaciones del sector salud y de seguros.

Este estudio usó el software Netlogo 6.o, un proyecto colaborativo de NorthWestern University, para implementar y tratar por medio de un modelo de simulación la complejidad de la emergencia de rutinas organizacionales, es decir el macro-nivel, a partir de las acciones de ejecución de tareas de los individuos del sistema, es decir el micro-comportamiento, incluyendo su exposición a un flujo de información que representa conocimiento del entorno.

Netlogo ha sido referido como un entorno de software para modelamiento de sistemas basados en agentes (MSBA), junto con Repast, Swarm y MASON (Fioretti, 2013; Li, Mao, Zeng, \& Wang, 2008; Tisue \& Wilensky, 2004).

Como medio de validación se recurrió a las pruebas de campo con expertos en las organizaciones seleccionadas, de conformidad con los ciclos de validación basada en el diseño.

\section{Configuración de la Capacidad de Absorción Organizacional}

Un estudio previo (Lopez-Cruz \& Obregon Neira, 2016) identifica al conocimiento como el elemento principal involucrado en la dinámica organizacional, confirmando que los elementos intangibles son los principales para generar identidad organizacional y ventaja competitiva sostenible (Calix, Vigier, \& Briozzo, 2015). Con el fin de aprovechar esta ventaja, generar un simulador por ordenador y evitar las fallas de modelos previos, se propone el re-diseño del constructo de la ACAP. Mientras que algunos modelos de la ACAP (Cohen \& Levinthal, 1990; Todorova \& Durisin, 2007) carecen de una especificación explícita de las interrelaciones de las organizaciones y su entorno, el diseño que presenta esta investigación considera elementos ambientales como determinante de los componentes del constructo. Además, la meta de este nuevo modelo no es describir sino prescribir la forma como las organizaciones pueden implementar ACAP. Esto se hace suministrando a las organizaciones una ayuda para diseñar sus propios medios para identificar las interacciones entre ellas (las organizaciones) y su entorno de donde se toma el conocimiento.

En este rediseño, se identificaron varias rutinas organizacionales como elementos que causan que el conocimiento fluya y sea atrapado o no. A partir de esto, las rutinas 
se organizan en clases o categorías: Identificación y reconocimiento, adquisición, asimilación, adopción/adaptación, aceptación, transformación, explotación e innovación. Finalmente, son ensambladas en un único artefacto por medio de flujos de conocimiento (ambiental) (figura 1).

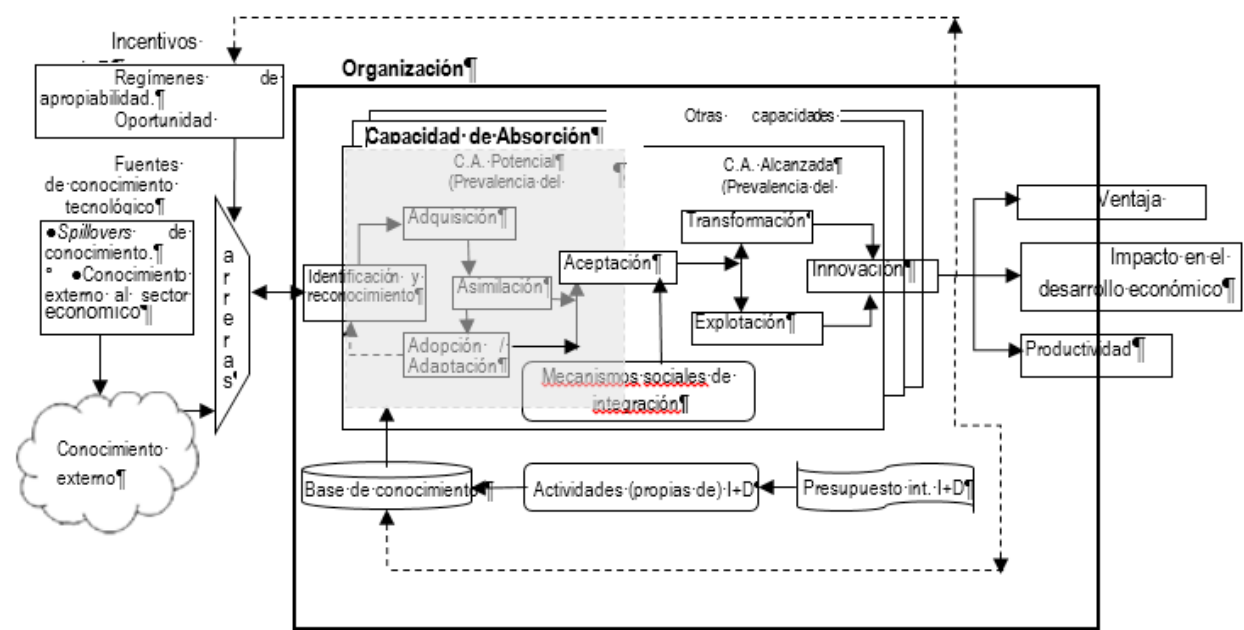

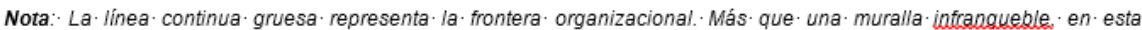

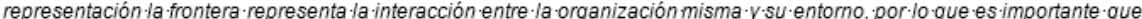

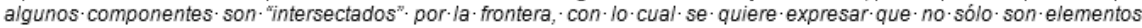

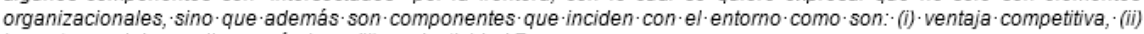

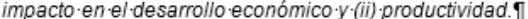

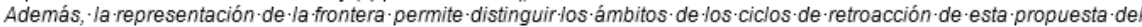
diseño $d e / \cdot$ constructo. $\uparrow$

Figura 1 - Un re-diseño de la capacidad de absorción (ACAP).

\subsection{Información, conocimiento y ACAP.}

El conocimiento es diferente de información. El conocimiento puede ser obtenido únicamente por medio de procesos de cognición e interpretación (Tecuci, 1992). Las organizaciones de aprendizaje adquieren conocimiento por medio de aprendizaje adaptativo -asumiendo cambios ambientales y haciendo cambios menores en los modelos mentales (paradigmas) y vía aprendizaje generativo -que es creativo y produce cambios en el modelo "mental" organizacional (Senge, 1990). El conocimiento en las organizaciones se verifica en relación con las estructuras y rutinas organizacionales, dado que no se deriva de la información disponible en un miembro en particular de la organización pero emerge como una propiedad del sistema de aprendizaje materializado en las personas (i.e. capital humano) (Rodríguez, Aguirre, \& Ordóñez, 2015). Además, está formado por la interacción entre diferentes procesos de aprendizaje que integrados a la organización (G. Dosi \& Marengo, 1993).

Elconocimientotácito estáincorporadoparcialmenteen hábitosyrutinasorganizacionales. Esto hace que el conocimiento tácito sea irreducible a información (Tecuci, 1992) y que no pueda ser transmitido en forma codificada. Por otro lado, los agentes económicos 
dependen del conocimiento tácito (Bareiss, Porter, \& Murray, 1989) y, por su parte, todas las habilidades y decisiones dependen del conocimiento tácito. De aquí la importancia de tener un constructo que permita una implementación más real y concreta.

El diseño del constructo que se propone aquí (Figura 1) reconoce la ACAP como un constructo complejo (Morin, 2011). La ACAP está integrada por componentes distintos, pero que interactúan mutuamente, que a su turnole permiten a la organización interactuar con su entorno. Sus componentes son rutinas (ACAP potenciales y alcanzadas) para identificar y reconocer el valor del conocimiento del entorno organizacional, así como rutinas para asimilación, adopción/adaptación, aceptación, transformación, explotación e innovación (Figura 1).

En este modelo, la ACAP se reconoce como una capacidad dinámica (una de muchas capacidades dinámicas de la organización). Este diseño retoma la distinción entre la ACAP potencial para representar el dominio donde la acción individual prevalece, y la ACAP alcanzada, donde prevalece la acción y el conocimiento colectivo prevalece sobre el individual.

El rol de las interacciones con el entorno (Figura 1) -algunos de ellos con información de retroacción como resultado de la transformación del conocimiento y la explotación- es capturar la alteración real de los regímenes de apropiación, debidos a la explotación del conocimiento, por medio de patentes o copyrights sobre el conocimiento que ha sido creado internamente. Además, este diseño captura la retroacción interna de la base de conocimiento que es alterada por el propio presupuesto de I+D (presupuesto interno) y es alterado actividades de $\mathrm{I}+\mathrm{D}$ internas, si existen.

La contribución principal del re-diseño de este constructo, que aquí se presenta, es la factibilidad de operacionalización cuando se implementa (para implementar) cada conjunto de rutinas organizacionales. Más aún, este diseño incluye varios ciclos de retroacción (líneas punteadas de derecha a izquierda en la Figura 1), que representan distintos elementos de conocimiento que alteran la ACAP en sí misma (como el eje dirigido que cruza desde 'adopción/adaptación' a 'identificación y reconocimiento') o dentro de la frontera de la organización (como el eje dirigido desde la 'innovación' hasta la 'base de conocimiento') o la organización y su entorno (como el eje dirigido desde 'innovación' hasta' incentivos de I+D. hasta donde se sabe, ningún otro modelo está más cerca de representar la naturaleza dinámica de la ACAP y sus ciclos de retroacción, que este modelo que se presenta aquí. Esto puede facilitar el desarrollo de modelos computacionales de simulación.

\subsection{Implementación de la ACAP.}

El modelo computacional de la ACAP fue implementado en NetLogo 6.0.1 (Wilensky, 1999) sobre sistema operacional Windows $10^{\circledR}$. Una rutina organizacional se implementa como una lista ordenada de tareas 1 hasta $n, n \in N$ (Miller, Pentland, \& Choi, 2012). La rutina organizacional consiste de una k-upla de tareas $(1 \leq \mathrm{k} \leq \mathrm{n})$. Sea $\mathrm{t}_{\mathrm{i}}(1 \leq \mathrm{i}<\mathrm{j}<\mathrm{k}<\mathrm{l} \leq \mathrm{m}$; $\mathrm{i}, \mathrm{m} \in \mathrm{N}$ ) una unidad mínima de acciones. La lista de tareas $\left[\mathrm{t}_{\mathrm{i}}, \mathrm{t}_{\mathrm{j}}, \mathrm{t}_{\mathrm{k}}, \mathrm{t}_{1}\right]$ es una rutina organizacional integrada por cuatro tareas: $t_{i}, t_{j}, t_{k}, t_{1} ; i<j<k<l$. En general, cada agente en el flujo de conocimiento exhibe una rutina organizacional (una lista) [t1, t2,... tn] donde $n$ es el tamaño de la lista. 


\section{Resultados and análisis}

Esta implementación facilitó la experimentación con diferentes configuraciones de la ACAP de una organización. Cada ejecución podría concluir en diferentes resultados, pero la relevancia era la obtención de distintos comportamientos como resultado de un cambio de uno o más parámetros iniciales (deslizadores de NetLogo) que controlan el flujo de conocimiento y de barreras. Además, el parámetro 'tiempo de vida' (ciclo de vida) tuvo sentido para los usuarios para representar distintos ciclos de negocios de diferentes productos de ICT. También, el 'tiempo de vida' permitió representar la obsolescencia del 'conocimiento' en las organizaciones. Esto es usualmente el caso, cuando, por ejemplo, una persona sabe cómo usar sistemas operacionales con líneas de comando como UNIX, iRMX, CP/M o MS-DOS y luego deben cambiar a sistemas operacionales con interface gráfica tipo GUI (Graphical User Interface).

Cuando los usuarios se concentraron en adquirir artefactos tecnológicos basados en conocimiento, bajo algunas circunstancias la cantidad de 'personas con conocimiento' en el flujo de conocimiento disminuyó

En algunas ejecuciones del simulador, las 'personas con conocimiento' en el flujo de conocimiento desaparecieron del todo. Los expertos explicaron esto como el resultado de la obsolescencia del conocimiento que las personas poseen y no existe una 'actualización' del conocimiento dentro de las organizaciones. Este es un resultado posible debido a que este modelo no representa organizaciones como universidades, institutos tecnológicos o centros de investigación. Un resultado simétrico aparece cuando el usuario se enfoca en reclutar 'personas con conocimiento' del flujo externo a la organización. Esto es un contexto extremo donde no hay industria tecnológica o comercio internacional. Así se genera un rezago tecnológico para las organizaciones y su vecindario o sector económico contextual.

Además de representar un rezago tecnológico, también representa la ausencia de políticas de investigación y desarrollo (organizacionales o nacionales) que tiendan a crear incentivos para la creación de nuevos productos (o servicios) que se puedan incorporar al flujo de conocimiento, lo que es otra forma de llamar a la innovación.

De acuerdo con los usuarios expertos, el ciclo de vida del conocimiento y el enfoque 'balanceado' en la adquisición de artefactos tecnológicos y 'personas con conocimiento' no garantiza el éxito de la organización en su contexto económico, que al parecer fue el comportamiento del modelo computacional en algunas situaciones. Además, un exceso en las barreras de conocimiento puede degradar el flujo de conocimiento en forma temprana.

\section{Conclusiones}

La creación de un modelo por ordenador que permita evaluar la configuración dinámica de la ACAP con base en el constructo ACAP ha permitido la validación funcional de esta capacidad dinámica en términos conceptuales de (i) identificar, (ii) asimilar y (iii) explotar el conocimiento disponible en el entorno (Cohen \& Levinthal, 1990). El modelo de simulación que se implementó en NetLogo (Wilensky, 1999) también facilitó la observación del usuario experto sobre la forma como sus distintas decisiones de acceder al flujo externo de conocimiento, crearon ventaja competitiva y mejoraron la productividad organizacional. 
Como consecuencia de lo anterior, una contribución de esta investigación es la propuesta de un re-diseño de la ACAP (figura 1), con ciclos de retroacción (feedback) externos e internos, en el que el conocimiento organizacional se representa mediante rutinas organizacionales y estas a su vez se implementan con una estructura de datos subyacente a un sistema basado en agentes por ordenador.

El segundo aporte de esta investigación es la evidencia de que el constructo de la ACAP es implementable en un modelo en ordenador basado en agentes no sólo para comprobar resultados estadísticos históricos, sino para propósitos de indagación y afinación de decisiones de diseño, cuyo beneficio es la capacidad de asistir en la prescripción de los componentes del constructo.

\section{Investigación futura}

Hay varias oportunidades para continuar el desarrollo de estos resultados. En primer lugar, puede realizarse una evaluación de la completitud del re-diseño de la ACAP con el fin de determinar si este constructo representa todas las rutinas organizacionales con las que cuentan las organizaciones innovadoras.

Además, un desarrollo computacional adicional puede ser conducido para enriquecer el modelo incluyendo el rol de las universidades y centros de investigación para 'reproducir' el conocimiento "incorporado" en la gente y en los artefactos tecnológicos que eventualmente fluyen dentro del flujo de conocimiento al que se exponen las organizaciones.

Laimplementación derutinas organizacionales puedeampliarseparaincluir otras categorías conceptuales que las entienden a ellas como (i) conjunto de reglas o procedimientos estándar y (ii) como tendencias que se vinculan a comportamientos previamente adquiridos que pueden ser 'activados' por los estímulos y contextos apropiados.

\section{Referencias}

Alexandre, C., \& Balsa, J. (2018). Um Sistema Multiagente no Combate ao Braqueamento de Capitais RISTI-Revista Ibérica de Sistemas e Tecnologias de Informação (25), 1-17.

Argyris, C., \& Schön, D. A. (1978). Organizational learning: A theory of action perspective (Vol. 173). Reading, MA, USA: Addison-Wesley.

Bareiss, E. R., Porter, B. W., \& Murray, K. S. (1989). Supporting strat-to-finish development of knowledge bases. Machine Learning, 4, 259-283.

Bobrow, D. G., \& Winograd, T. (1977). An overview of KRL, a knowledge representation language. Cognitive Science, 1(1), 3-45.

Calix, C. G., Vigier, H. P., \& Briozzo, A. E. (2015). Capital intelectual y otros determinantes de la ventaja competitiva en empresas exportadoras de la zona norte de Honduras. Suma de Negocios, 6(14), 130-137.

Cohen, W. M., \& Levinthal, D. A. (1989). Innovation and learning: the two faces of R \& D. The Economic Journal, 569-596. 
Cohen, W. M., \& Levinthal, D. A. (1990). Absorptive capacity: a new perspective on learning and innovation. Administrative science quarterly, (35), 128-152.

Cohen, W. M., \& Levinthal, D. A. (1994). Fortune favors the prepared firm. Management Science, 4O(2), 227-251.

Cordes-Berszinn, P. (2013). Dynamic capabilities: how organisational structures affect knowledge processes. Berlin: Springer.

Christensen, C. M., \& Overdorf, M. (2000). Meeting the challenge of disruptive change. Harvard business review, 78(2), 66-77.

Dhanaraj, C., Lyles, M. A., Steensma, H. K., \& Tihanyi, L. (2004). Managing tacit and explicit knowledge transfer in IJVs: the role of relational embeddedness and the impact on performance. Journal of International Business Studies, 35(5), 428-442.

Donoso Barraza, Í., \& Vega Zepeda, V. (2017). Factores sociales y humanos que afectan el proceso de educción de requerimientos: una revisión sistemática. RISTI-Revista Ibérica de Sistemas e Tecnologias de Informação, (24), 69-83.

Dosi, G., \& Marengo, L. (1993). Toward an evolutionary theory of organizational competencies. In R. W. England (Ed.), Evolutionary concepts in contemporary economics. Ann Arbor: University of Michigan Press.

Dosi, G., \& Marengo, L. (2000). On the tangled discourse between transaction costs economics and competence-based views of the firms: Some comments. Competence, governance, and entrepreneurship: Advances in economic strategy research, 80-92.

Dozier, K., \& Chang, D. (2009). The role of information technology in managing supply chain fluctuations. Paper presented at the WSEAS Management, Marketing and Finances conference in Houston.

Easterby-Smith, M., Graca, M., Antonacopoulou, E., \& Ferdinand, J. (2005). Absorptive capacity in practice: an empirical examination of Zahra and George's model. retrieved from: https://pdfs.semanticscholar. org/3230/o8710fed3bf250adoo42cf3360e2690133de.pdf

Eisenhardt, K. M., \& Martin, J. A. (2000). Dynamic capabilities: What are they. Strategic management journal, 21(1), 1105-1121.

Eisenhardt, K. M., \& Santos, F. M. (2002). Knowledge-based view: A new theory of strategy. Handbook of strategy and management, 1, 139-164.

Fabrizio, K. R. (2009). Absorptive capacity and the search for innovation. Research policy, 38(2), 255-267.

Filgueiras Sainz de Rozas, M. L., Castro Fernández, M., \& Rafull Suárez, I. (2013). Determinación de la capacidad de absorción: estudio de caos en la empresa GEYSEL. Ingeniería Energética, XXXIV(3), 175-185.

Fioretti, G. (2013). Agent-based simulation models in organization science. Organizational Research Methods, 2013(journals.sagepub.com). 
González, I. (2014). Modelo teórico de capacidad de absorción, innovación organizacional y emprendimiento. Informe de Investigaciones Educativas, 27(1), 290-340.

Grant, R. M. (1996). Toward a knowledge-based theory of the firm. Strategic management journal, $17(\mathrm{~S} 2), 109-122$.

Grimm, V., Berger, U., Bastiansen, F., Eliassen, S., Ginot, V., Giske, J., \& Huse, G. (2006). A standard protocol for describing individual-based and agent-based models. Ecological modelling, 198(1), 115-126.

Helfat, C. E., Finkelstein, S., Mitchell, W., Peteraf, M., Singh, H., Teece, D., \& Winter, S. G. (2009). Dynamic capabilities: Understanding strategic change in organizations: Hoboken: John Wiley \& Sons.

Hevner, A., March, S. T., Park, J., \& Ram, S. (2004). Design science in information systems research. MIS quarterly, 28(1), 75-105.

Hevner, A. R., \& Chatterjee, S. (2010). Design research in information systems : theory and practice. New York, London: Springer.

Huber, G. P. (1991). Organizational learning: The contributing processes and the literatures. Organization science, 2(1), 88-115.

Klügl, F. (2008). A validation methodology for agent-based simulations. In Proceedings of the 2008 ACM symposium on Applied computing. Fortaleza, Ceara, Brazil: ACM.

Lane, P. J., Koka, B. R., \& Pathak, S. (2006). The reification of absorptive capacity: A critical review and rejuvenation of the construct. Academy of management review, 31(4), 833-863.

León-Sigg, M. d., Vázquez-Reyes, S., \& Villa-Cisneros, J. L. (2017). Factores que afectan la adopcion de Tecnologias de Informacion en micro y pequenas empresas: un estudio cualitativo. RISTI-Revista Ibérica de Sistemas e Tecnologias de Informação, (22), 20-36.

Levitt, B., \& March, J. G. (1988). Organizational learning. Annual review of sociology, 14, 319-340.

Lewin, A. Y., Massini, S., \& Peeters, C. (2011). Microfoundations of internal and external absorptive capacity routines. Organization science, 22(1), 81-98.

Li, X., Mao, W., Zeng, D., \& Wang, F.-Y. (2008). Agent-based social simulation and modeling in social computing. Paper presented at the International Conference on Intelligence and Security Informatics. Taipei, Taiwan.

López-Cruz, O. (2010). Transferencia de tecnología informática: entorno colombiano. Revista de Tecnología - Journal of Technology, 9(1), 19-23.

Lopez-Cruz, O., \& Obregon Neira, N. (2016). Diseño de la capacidad de absorción en las organizaciones: propuesta de un nuevo constructo y literatura. Paper presented at the Congreso Nacional e Internacional en Innovación en la Gestión de Organizaciones, Abril, 2016, Bogotá. 
López-Paniagua, I., Nieto-Carlier, R., Rodríguez-Martín, J., González-Fernández, C., \& Jiménez-Álvaro, Á. (2011). Clases prácticas: Una herramienta esencial en la enseñanza de las ingenierías en el marco del Espacio Europeo de Educación Superior. Dyna, 86(5), 523-530.

March, S. T., \& Storey, V. C. (2008). Design science in the information systems discipline: an introduction to the special issue on design science research. MIS quarterly, 32(4), 725-730.

Marín, A., Laureiro, D., \& Forero, C. (2007). Innovation patterns and intellectual property in SMEs of a developing country. Galeras de Administración, 17.

Miller, K. D., Pentland, B. T., \& Choi, S. (2012). Dynamics of performing and remembering organizational routines. Journal of Management Studies, 49(8), 1536-1558.

Morin, E. (2011). Introducción al pensamiento complejo. Barcelona, España: Gedisa.

Müller, B., Bohn, F., Dreßler, G., Groeneveld, J., Klassert, C., Martin, R., \& Schwarz, N. (2013). Describing human decisions in agent-based models-ODD+ D, an extension of the ODD protocol. Environmental Modelling \& Software, 48, 37-48.

Niazi, M. A., Hussain, A., \& Kolberg, M. (2009). Verification and validation of agent based simulations using the VOMAS (virtual overlay multi-agent system) approach. Paper presented at the MAS\&S at Multi-Agent Logics, Languages, and Organisations Federated Workshops (MALLOW), Turin, Italy, September 7-10, 2009.

Nickerson, J. A., \& Zenger, T. R. (2004). A knowledge-based theory of the firm-The problem-solving perspective. Organization science, 15(6), 617-632.

Nishiyama, T., Ikeda, K., \& Niwa, T. (2000). Technology Transfer Macro-Process: A Practical Guide for the Effective Introduction of Technology. Paper presented at the ICSE 2000, Limerick Ireland, June 4-11, 2000.

Nonaka, I., \& Takeuchi, H. (1995). The knowledge-creating company : how Japanese companies create the dynamics of innovation. New York: Oxford University Press.

Nonaka, I., Toyama, R., \& Nagata, A. (2000). A firm as a knowledge-creating entity: a new perspective on the theory of the firm. Industrial and corporate change, 9(1), 1-20.

Palos-Sánchez, P. R., Arenas-Márquez, F. J., \& Aguayo-Camacho, M. (2017). La adopción de la tecnología cloud computing (SaaS): efectos de la complejidad tecnológica vs formación y soporte. RISTI-Revista Ibérica de Sistemas e Tecnologias de Informação, (22), 89-105.

Parunak, H. V. D., Savit, R., \& Riolo, R. L. (1998). Agent-based modeling vs. equationbased modeling: A case study and users' guide. Paper presented at the International Workshop on Multi-Agent Systems and Agent-Based Simulation, Sao Paulo, Brazil.

Porter, M. E. (1998). Competitive strategy : techniques for analyzing industries and competitors : with a new introduction. New York: Free Press.Traducción a castellanoEstrategia Competitiva : Técnicas Para El análisis De Los Sectores Industriales y De La Competencia. México D.F.: Compañía Editorial Continental, 1998. 
Prahalad, C. K., \& Hamel, G. (2006). The core competence of the corporation. Berlin: Springer.

Rodríguez, A. S. D., Aguirre, R. C., \& Ordóñez, L. S. (2015). Clima organizacional, migración tecnológica y apagón analógico en la televisión ecuatoriana. . Suma de Negocios, 6(14), 138-146.

Sankararaman, S., \& Mahadevan, S. (2015). Integration of model verification, validation, and calibration for uncertainty quantification in engineering systems. Reliability Engineering \& System Safety, 138, 194-209.

Senge, P. (1990). The Fifth Discipline: The Art \& Practice of The Learning Organization. New York: Double Day Dell Publisher.

Simon, H. A. (1996). The sciences of the artificial (Vol. 136). Cambridge, MA, USA: MIT press.

Sousa, N. M., Costa, C. J., \& Aparicio, M. (2017). Ba: um fator determinante no uso de sistemas de gestão do conhecimento. RISTI-Revista Ibérica de Sistemas e Tecnologias de Informação, (22), 1-19.

Tecuci, G. (1992). Cooperation in knowledge base refinement. Paper presented at the Machine Learning: Proceedings of the Ninth International Conference ML92.

Teece, D., \& Pisano, G. (1994). The dynamic capabilities of firms: an introduction. Industrial and corporate change, 3(3), 537-556.

Teece, D. J. (1977). Technological transfer by mutlinational firms: the resource cost of transferring know-how. Economic Journal, 87(June), 242-261.

Teece, D. J., Pisano, G., \& Shuen, A. (1997). Dynamic capabilities and strategic management. Strategic management journal, 18(7), 509-533.

Tisue, S., \& Wilensky, U. (2004). NetLogo: A Simple Environment for Modeling Complexity. Paper presented at the International Conference on Complex Systems, May 16-21, Boston.

Todorova, G., \& Durisin, B. (2007). Absorptive capacity: valuing a reconceptualization. Academy of management review, 32(3), 774-786.

Vega, J., Gutiérrez, A., \& Fernández de Lucio, I. (2007). An analytical model of absorptive capacity. Paper presented at the DRUID Summer Conference, Copenhagen, 18-20 June.

Volberda, H. W., Foss, N. J., \& Lyles, M. A. (2009). Absorbing the Concept of Absorptive Capacity: How To Realize Its Potential in the Organization Field.

Wilensky, U. (1999). NetLogo. http://ccl.northwestern.edu/netlogo/. Center for Connected Learning and Computer-Based Modeling. Evanston, IL: Northwestern University.

Winter, S. G. (2000). The satisficing principle in capability learning. Strategic management journal, 21(10-11), 981-996. 
Xiang, X., Kennedy, R., Madey, G., \& Cabaniss, S. (2005). Verification and validation of agent-based scientific simulation models. Paper presented at the Agent-Directed Simulation Conference, San Diego, CA, USA.

Zahra, S. A., \& George, G. (2002). Absorptive capacity: A review, reconceptualization, and extension. Academy of management review, 27(2), 185-203.

Zollo, M., \& Winter, S. G. (2002). Deliberate learning and the evolution of dynamic capabilitiesv. Organization science, 13(3), 339-351. 
